\title{
Starch Trek: The Search for Yield
}

\author{
James R. Lloyd and Jens Kossmann* \\ Department of Genetics, Institute for Plant Biotechnology, University of Stellenbosch, Stellenbosch, South Africa
}

Starch is a plant storage polyglucan that accumulates in plastids. It is composed of two polymers, amylose and amylopectin, with different structures and plays several roles in helping to determine plant yield. In leaves, it acts as a buffer for night time carbon starvation. Genetically altered plants that cannot synthesize or degrade starch efficiently often grow poorly. There have been a number of successful approaches to manipulate leaf starch metabolism that has resulted in increased growth and yield. Its degradation is also a source of sugars that can help alleviate abiotic stress. In edible parts of plants, starch often makes up the majority of the dry weight constituting much of the calorific value of food and feed. Increasing starch in these organs can increase this as well as increasing yield. Enzymes involved in starch metabolism are well known, and there has been much research analyzing their functions in starch synthesis and degradation, as well as genetic

OPEN ACCESS

Edited by:

Miguel Angel Ballicora, Loyola University Chicago, United States

Reviewed by:

Robert D. Hancock, James Hutton Institute, United Kingdom Nishikant Wase, University of Nebraska-Lincoln, United States

*Correspondence: Jens Kossmann kossmann@sun.ac.za

Specialty section:

This article was submitted to Plant Metabolism and Chemodiversity, a section of the journal Frontiers in Plant Science

Received: 19 September 2018 Accepted: 12 December 2018

Published: 21 January 2019

Citation:

Lloyd JR and Kossmann J (2019) Starch Trek: The Search for Yield.

Front. Plant Sci. 9:1930.

doi: 10.3389/fpls.2018.01930 and posttranslational regulatory mechanisms affecting them. In this mini review, we examine work on this topic and discuss future directions that could be used to manipulate this metabolite for improved yield.

Keywords: starch, sucrose, carbohydrate partitioning, photosynthesis, metabolism

\section{INTRODUCTION}

The need for improved crop yields due to an increase in world population and a decrease in available agricultural land is well known (Edgerton, 2009). This problem will likely be exacerbated through alterations in environmental conditions caused by anthropomorphic $\mathrm{CO}_{2}$ release that may lead to increases in both biotic and abiotic stresses (Fodor et al., 2017). There are many potential biotechnological methods that can lead to increased yield, and one of these involves altering starch metabolism. This mini review will examine work that has been performed to improve plant yield through manipulation of this metabolite and suggest new avenues that could be explored.

Starch is a polyglucan that is stored as granules within plastids. It consists of two polymers with differing structures, amylose and amylopectin. Amylose contains relatively long (normally composed of several hundred glucose monomers) $\alpha 1,4$ linked chains, while amylopectin is composed of many short (approximately 5-50 glucose monomers) $\alpha 1,4$ linked chains, linked together by $\alpha 1,6$ branch points in an ordered, crystalline array (Zeeman et al., 2010).

Manipulation of starch metabolism is important for improving plant yield for several reasons. As starch is the major form of calories within plants, increasing starch concentrations in plant tissues can mean that less food or fodder has to be consumed to supply the same energetic value (Ruckle et al., 2017). It is also a major sink within storage organs, so increasing starch here can lead to increased plant yield simply as more accumulates. In leaves, it plays two roles. Firstly, it is synthesized during the day and degraded at night, buffering the plant from night time carbon starvation (Stitt and Zeeman, 2012; Arias et al., 2014) which leads 
to transcriptional upregulation of stress-related genes (Stitt et al., 2007) and an inhibition of gibberellin synthesis (Paparelli et al., 2013). Secondly, it is a source of carbon skeletons for the production of compatible solutes that help plants to overcome abiotic stress (Thalmann and Santelia, 2017). Finally, starch has been shown to affect developmental processes (Matsoukas et al., 2013), and therefore, its manipulation could increase yield through altering plant or seed development.

\section{Starch Metabolism}

Due to its many roles, the pathway of starch metabolism has been studied intensively, and many enzymatic steps involved in its metabolism have been elucidated. This knowledge has been used to construct a detailed model of its metabolism, which has been described in several recent reviews (Bahaji et al., 2014; Pfister and Zeeman, 2016; MacNeill et al., 2017). The rest of this section will briefly outline the major enzymes involved to allow for further discussion of the manipulation of the pathway.

Starch polymer formation (Figure 1B) involves the synthesis of adenosine diphosphate (ADP)-glucose by ADP-glucose pyrophosphorylase (AGPase). This is used by starch synthases (SS) to form linear $\alpha 1,4$ glucan chains. One starch synthase isoform is responsible for amylose synthesis, while several others are involved in granule initiation and amylopectin synthesis (Pfister and Zeeman, 2016; Nazarian-Firouzabadi and Visser, 2017). The branch points within amylopectin are introduced by starch branching enzyme (SBE) isoforms, while excess $\alpha 1,6$ links are removed by isoamylases (Pfister and Zeeman, 2016; MacNeill et al., 2017).

Starch degradation (Figure 1A) is initiated by glucan water dikinase (GWD) enzymes that phosphorylate the granule, helping to solubilize it and allow access to $\alpha$-, $\beta$-, and isoamylases (Streb et al., 2012; Mahlow et al., 2016). These release phosphorylated malto-oligosaccharides into the stroma, where the phosphate is removed by glucan phosphatases, allowing further degradation to maltose and glucose by the combined actions of amylases and disproportionating enzyme 1. Maltose and glucose are exported to the cytosol through specific transport proteins, where maltose becomes further mobilized by disproportionating enzyme 2 (DPE2; Lloyd and Kossmann, 2015).

The regulation of starch metabolism takes place at several levels, including both transcriptional and posttranslational mechanisms. A number of transcription factors affecting expression of genes encoding starch metabolizing enzymes have been identified, and their roles are currently being examined. At the posttranslational level, allosteric regulation (Zeeman et al., 2010) protein phosphorylation (Kötting et al., 2010) and reducing/oxidation (redox) conditions (Santelia et al., 2015; Skryhan et al., 2018) are known to influence both enzyme activities and the formation of enzyme complexes. The roles of these processes will probably differ between leaves and heterotrophic storage organs. For example, the redox status within chloroplasts varies over a day/night cycle, where reducing condition predominates during the day and oxidizing conditions at night. Within amyloplasts of heterotrophic tissues, this day/ night cycle will not occur, although it has been shown that the alteration in redox status of chloroplasts can be sensed by amyloplasts (Balmer et al., 2006). Finally, nutrient sensing mechanisms would be expected to link the accumulation of soluble sugars, such as sucrose, with starch synthesis through a combination of mechanisms including 14-3-3 proteins, hexokinase (Rolland et al., 2006), SnRKs (Crozet et al., 2014; Wurzinger et al., 2018), and TOR kinases (Rolland et al., 2006; Dobrenel et al., 2016; Shi et al., 2018).

\section{ADP-Glucose Pyrophosphorylase-A Key Enzyme for Yield in Both Storage Organs and Leaves}

ADP-glucose pyrophosphorylase (Figure 1B) has been shown to be a key enzyme influencing starch accumulation in both leaves and storage organs. One complicating factor is the level of posttranslational control of the enzyme by a combination of allosteric effectors and redox, which means that overexpression of the native enzyme may not increase flux into starch synthesis (Boehlein et al., 2013a). This has been overcome through the use of mutated sequences encoding allosteric and redox insensitive isoforms (Tuncel and Okita, 2013). In cereal endosperm, another complication is that most AGPase activity is found within the cytosol, with a specific transporter localized in the outer plastid membrane importing ADP-glucose into the stroma, while in noncereal species it is found only in the plastid. Within cereal endosperm, the cytosolic pathway plays a greater role than the plastidial one (Tuncel and Okita, 2013; Tetlow and Emes, 2017).

It has been known for many years that increasing AGPase activity leads to increased starch synthesis, and as was mentioned above, increasing starch in food and fodder crops would improve their calorific value (Ruckle et al., 2017, 2018). However, interestingly, increasing starch amounts in this way can have beneficial effects on plant productivity. In Arabidopsis and rice, where leaf AGPase activity has been upregulated, leaf starch amounts are increased at the end of the day and the plants grow larger (Gibson et al., 2011; Schlosser et al., 2014; Oiestad et al., 2016), most likely caused by elevated nighttime sugar levels (Stitt and Zeeman, 2012; Arias et al., 2014).

Increasing AGPase in storage organs can also increase starch amounts (Zeeman et al., 2010; Sonnewald and Kossmann, 2013; Tuncel and Okita, 2013). Initial work utilized a bacterial gene that was insensitive to allosteric regulation (Stark et al., 1992); however, more recent work has used plant genes engineered to encode proteins with improved properties (Tuncel and Okita, 2013). Due to the dual cytosolic and plastidial localization of AGPase in cereal endosperm (Figure 1B) compared to its plastidial targeting in other organs and species, the subcellular targeting of AGPase is critical for successful upregulation of starch synthesis. In noncereal species, transgenic plants with increased plastidial AGPase activity accumulate increased amounts of starch, while in cereal endosperm the enzyme has to be localized to the cytosol to have an effect (Zeeman et al., 2010; Sonnewald and Kossmann, 2013; Tuncel and Okita, 2013).

It is clear that AGPase plays a critical role in determining starch yield. It is important to understand, therefore, how its properties could be altered to increase yield, especially 


\section{A}
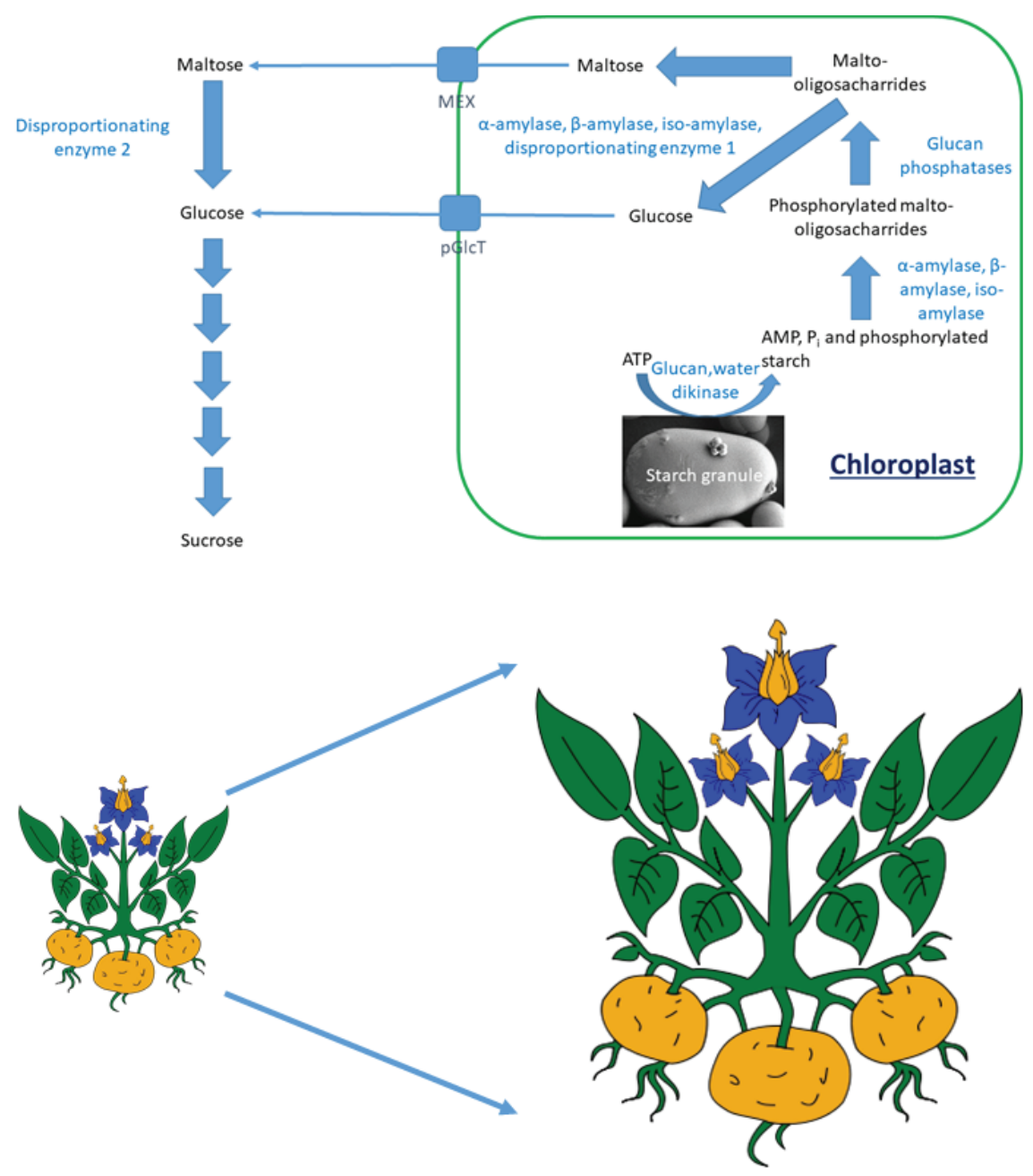

B

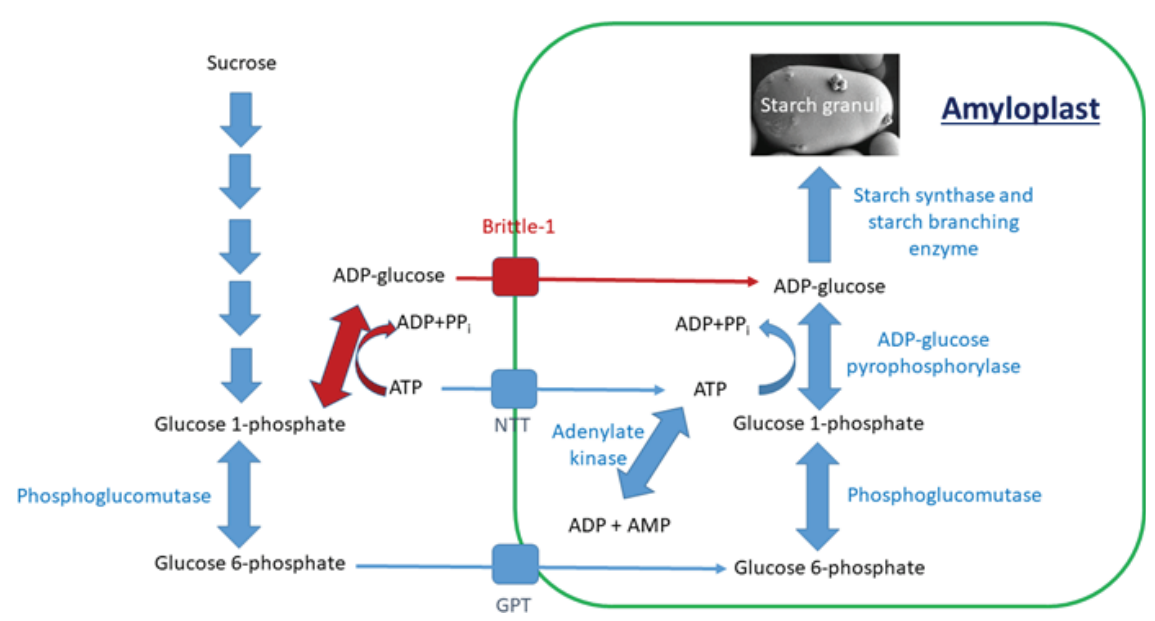

FIGURE 1 | The pathway of starch degradation in (A) leaves and of starch synthesis in (B) storage organs. (A) Starch is degraded through a series of enzymatic steps to maltose and glucose. These are transported from the chloroplast by either the maltose export (MEX) or plastidial glucose transport (pGlcT) proteins. Within the cytosol, they are converted to sucrose for further transport. (B) There are two pathways of starch synthesis in vascular plants. One is shared in all plants (blue arrows), while the other is present only in cereal endosperm (red arrows). Sucrose is degraded to glucose 6-phosphate, which is transported into amyloplasts by the glucose 6-phosphate/phosphate transporter (GPT). The ADP-glucose pyrophosphorylase step within the amyloplast utilizes ATP, which is imported into the amyloplast by the plastidial ATP/ADP transporter (NTT). Within cereal endosperm, ADP-glucose is synthesized extraplastidially and is imported into amyloplasts by the Brittle-1 transporter. 
in a changing environment. Protein engineering using plant genes has led to much knowledge about reducing redox or allosteric inhibition of its activity as well as improving its activity under heat stress (Georgelis and Hannah, 2008; Haedrich et al., 2012; Boehlein et al., 2013a,b, 2015), and many of these engineered proteins have been transferred into plants through transgenic technology (Tuncel and Okita, 2013). A recent TILLING population targeting this enzyme in Arabidopsis has been developed and may help in developing nontransgenic routes to increase starch synthesis in vivo using gene editing (Haedrich et al., 2011). Although increasing AGPase activity will increase flux into the pathway, this is not the only way that it increases yield. In several cereals, for example, over-expression of AGPase can increase seed number, which in maize has been shown to occur through an effect on maternal tissues (Smidansky et al., 2002, 2003; Hannah et al., 2012, 2017).

Another strategy used to influence the AGPase step in storage organs has been to increase supply of its substrates, adenosine triphosphate (ATP) and/or glucose 6-phosphate. In dicots, ATP limitation of AGPase within plastids has been suggested (Geigenberger, 2001), meaning that increasing supply would be a necessity to increase starch contents. This has been achieved in potato through overexpression of a plastidial adenylate translocator (NTT; which counter-exchanges ATP for ADP; Figure 1B). However, reports on the effect of increasing NTT expression on starch amounts are mixed. Two studies in potato found that this increased starch amounts (Tjaden et al., 1998; Geigenberger, 2001) while another described no alteration (Zhang et al., 2008). That later study did, however, find that combined overexpression of the NTT and a plastidial glucose 6-phosphate translocator leads to increased starch and tuber yield (Zhang et al., 2008).

A second strategy to influence ATP supply occurred through manipulation of a plastidial isoform of adenylate kinase, an enzyme that interconverts ATP with ADP and adenosine monophosphate (AMP) (Figure 1B). Repression of this enzyme led to increased ADP-glucose, tuber starch content, and yield (Regierer et al., 2002), presumably due to an increase in the plastidial ATP pool. Indeed, manipulation of nucleotide metabolism generally may be a profitable way to increase starch contents. In addition to the adenylate kinase study described above, repression of UMP synthase has been demonstrated to lead in uridine nucleotides accompanied by an increase in both cell wall and starch (Geigenberger et al., 2005). This is most likely caused by increases of flux into both components through increased sucrose degradation.

The work performed in altering substrate supply for AGPase has generally been performed in dicotyledonous plants. A recent study (Cakir et al., 2016) has examined rice plants where an increase in extra-plastidial AGPase activity was combined with overexpression of the plastidial ADP-glucose transporter (Figure 1B). They found that, although they could identify increased plastidial ADP-glucose amounts, this did not lead to an increase in starch, indicating additional stromal barriers affecting this pathway. If these could be identified, then they could be manipulated to allow increased starch accumulation.

\section{Can Alterations in Other Starch Biosynthetic Enzymes Increase Yield?}

Although most work has examined influencing AGPase as a method of increasing starch amounts, it may not be the only protein that can do this. The polymerizing enzymes SS and SBE (Figure 1B) are present as multiple isoforms, which often play differing roles in determining the structure of the amylopectin molecule. It has been reported that increased expression of SS encoding genes is associated with increased starch accumulation and grain weight caused by expression of a mutated ubiquitin receptor in maize (Xie et al., 2018), while overexpression of one SS isoform increased potato tuber starch content (Gámez-Arjona et al., 2011). The data of Gámez-Arjona et al. (2011) have, however, been questioned (Sonnewald and Kossmann, 2013) due to the lack of dry matter increase that accompanied the reported starch elevation. Nevertheless, these data indicate that manipulation of SS isoforms may be a profitable way in increasing starch content.

There are fewer reports of SBE overexpression; however, one study in potatoes demonstrated that this led to synthesis of starch with altered structure but did not report on an effect on yield (Brummell et al., 2015). Interestingly, manipulation of SBE activity in Arabidopsis leaves has revealed a potential method for improving growth. Replacement of endogenous Arabidopsis activities with two from maize led to plants with increased starch in their leaves and improved seed yield, most likely also due to increased night-time sugar levels (Liu et al., 2016).

\section{Are Starch Degradative Enzymes Useful for Yield Increases?}

Plant biotechnologists have generally attempted to increase starch yield through altering activities of enzymes involved in its synthesis. Degradative enzymes have often been ignored as targets for improving yield as mutations in them are often associated with reduced plant growth (Stitt and Zeeman, 2012; Paparelli et al., 2013). Recent work, however, has demonstrated that repression of the starch phosphorylating GWD enzyme (Figure 1A) in wheat endosperm improved both growth and seed production. Unfortunately, although this was demonstrated convincingly in glasshouse trials (Ral et al., 2012; Bowerman et al., 2016), when the same lines were examined in the field a reduction in yield was observed (Whan et al., 2017). Although it may not be a valuable way to increase starch yield in storage organs, repressing genes involved in this process could improve forage and silage crops by improving their calorific value for animal feed (Weise et al., 2012; Ruckle et al., 2018).

Manipulating starch degradation (Figure 1A) may have a greater role in helping plants overcome abiotic stress (Thalmann and Santelia, 2017). One of the main metabolites produced during starch catabolism is maltose, and it has been demonstrated that this can help stabilize photosynthetic membranes (Kaplan and Guy, 2004). dpe2 mutant plants, which accumulate maltose, demonstrate reduced freezing damage ( $\mathrm{Li}$ et al., 2011); however, they also grow smaller than wild-type plants (Chia et al., 2004). One strategy to overcome this may be the use of stress inducible promoters to drive repression constructs that reduce $D p e 2$ expression 
only at times when increased maltose would be advantageous. Simultaneously, $\beta$-amylases that produce maltose during starch degradation (Kaplan and Guy, 2004; Kaplan et al., 2006) could be upregulated to increase levels of this metabolite further.

\section{Control Mechanisms Affecting Starch Metabolism}

Although alterations of individual enzymatic steps within the starch pathway can have a beneficial effect on yield, alterations in transcriptional control mechanisms allow the possibility to influence these in a beneficial manner through altering multiple steps simultaneously. A few transcriptional regulators affecting starch metabolism have been identified (Zhang et al., 2005; Fu and Xue, 2010; Guan et al., 2011; Wang et al., 2013; Gontarek et al., 2016; Xiao et al., 2017), and manipulation of one of these led to increased seed size and yield in rice ( $\mathrm{Fu}$ and Xue, 2010). More recently, transcriptional analysis has led to the identification of genes putatively involved in regulating starch metabolic genes (see for example Van Harsselaar et al., 2017), but their roles have often not been studied in detail. Such functional analyses would help in identification of factors that could be used to improve yield.

Post-transcriptional regulation will also be influential in controlling starch metabolism. Many sugar sensing mechanisms involving 14-3-3- and SnRK proteins, trehalose metabolism, TOR kinases, and hexokinase are known to affect starch metabolism. Alterations in expression of some 14-3-3 and SnRK proteins can lead to improved starch accumulation or to the upregulation of enzymes involved in starch synthesis, in several species (Sehnke et al., 2001; McKibbin et al., 2006; Wang et al., 2016, 2018). Trehalose 6-phosphate (T6P) has been proposed to activate AGPase through a post-translational redox mechanism (Kolbe et al., 2005), while genetic manipulation of T6P amounts can increase starch in leaves through repression of starch degradation (Martins et al., 2013) as well as increase yield in maize (Nuccio et al., 2015). More recently, it has been demonstrated that application of plant permeable analogues of T6P increases endosperm starch content and yield in wheat. The reasons for this are not entirely clear as many transcriptional and metabolic changes were identified; however, application of the same analog to Arabidopsis increased AGPase activity which would provide a direct explanation for the increased starch (Griffiths et al., 2016).

Post-translational alteration of proteins involved in starch metabolism can involve protein phosphorylation (Kötting et al., 2010) or reduction/oxidation mechanisms (Glaring et al., 2012; Santelia et al., 2015). Little is known about protein phosphorylation influencing starch metabolism, although a recent paper has identified plastidially localized protein kinases and phosphatases that may interact with starch metabolic

\section{REFERENCES}

Arias, M. C., Pelletier, S., Hilliou, F., Wattebled, F., Renou, J.-P., and D'Hulst, C. (2014). From dusk till dawn: the Arabidopsis thaliana sugar starving responsive network. Front. Plant Sci. 5:482. doi: 10.3389/fpls.2014.00482

Bahaji, A., Li, J., Sánchez-López, Á. M., Baroja-Fernández, E., Muñoz, F. J., Ovecka, M., et al. (2014). Starch biosynthesis, its regulation and biotechnological enzymes (White-Gloria et al., 2018), which is a first step in the study of this process. More is known about redox control as several genes involved in starch metabolism are known to be redox regulated (Fu et al., 1998; Ballicora et al., 2000; Tiessen et al., 2002; Sokolov et al., 2006; Valerio et al., 2011; Glaring et al., 2012; Seung et al., 2013; Shaik et al., 2014), and expression of thioredoxin-f increased starch amounts in tobacco leaves (Sanz-Barrio et al., 2013), although it is not clear which enzymes were affected. The best characterization of the role of redox on starch metabolizing enzymes in vivo has been the examination of AGPase and GWD, where redox insensitive proteins have been expressed in plants (Haedrich et al., 2012; Skeffington et al., 2014). Redox insensitive AGPase led to increased leaf starch, although this was dependent on day length (Haedrich et al., 2012). On the other hand, constitutive expression of a redox insensitive GWD had little effect on leaf starch degradation (Skeffington et al., 2014), which agrees with the observation that reducing conditions (which would be expected to be present in chloroplasts during the day) activate the wild-type enzyme (Mikkelsen et al., 2005).

\section{Future Prospects}

Although much has been achieved over the past decades in the manipulation of starch metabolism, there are still improvements that can be made. Detailed analysis of both transcriptional and post-translational control mechanisms will help fine tune current attempts at manipulating the pathway. Perhaps most importantly is the rational integration of metabolic engineering simultaneously in leaves and storage organs. One attempt to accomplish this involved reducing starch synthesis in potato leaves in order to increase soluble sugar export, while simultaneously increasing substrate supply to starch synthesis in tubers through overexpression of two plastidial transport proteins (Jonik et al., 2012). This approach led to an increase over and above the amounts found when either leaf or tuber metabolism was altered alone, doubling starch yield. We believe that such integrated approaches will lead to the greatest benefit for crop improvement.

\section{AUTHOR CONTRIBUTIONS}

JL wrote the first draft and edited the manuscript alongside JK.

\section{FUNDING}

This work was supported by the NRF SARCHI chair "Genetic Tailoring of Biopolymers."

approaches to improve crop yields. Biotechnol. Adv. 32, 87-106. doi: 10.1016/j. biotechadv.2013.06.006

Ballicora, M. A., Frueauf, J. B., Fu, Y., Schurmann, P., and Preiss, J. (2000). Activation of the potato tuber ADP-glucose pyrophosphorylase by thioredoxin. J. Biol. Chem. 275, 1315-1320. doi: 10.1074/jbc.275.2.1315

Balmer, Y., Vensel, W. H., Cai, N., Manieri, W., Schurmann, P., Hurkman, W. J., et al. (2006). A complete ferredoxin/thioredoxin system regulates fundamental 
processes in amyloplasts. Proc. Natl. Acad. Sci. U. S. A. 103, 2988-2993. doi: 10.1073/pnas.0511040103

Boehlein, S. K., Shaw, J. R., Hwang, S. K., Stewart, J. D., and Curtis Hannah, L. (2013a). Deciphering the kinetic mechanisms controlling selected plant ADP-glucose pyrophosphorylases. Arch. Biochem. Biophys. 535, 215-226. doi: 10.1016/j.abb.2013.04.003

Boehlein, S. K., Shaw, J. R., McCarty, D. R., Hwang, S.-K., Stewart, J. D., and Hannah, L. C. (2013b). The potato tuber, maize endosperm and a chimeric maize-potato ADP-glucose pyrophosphorylase exhibit fundamental differences in Pi inhibition. Arch. Biochem. Biophys. 537, 210-216. doi: 10.1016/j. abb.2013.07.019

Boehlein, S. K., Shaw, J. R., Stewart, J. D., Sullivan, B., and Hannah, L. C. (2015). Enhancing the heat stability and kinetic parameters of the maize endosperm ADP-glucose pyrophosphorylase using iterative saturation mutagenesis. Arch. Biochem. Biophys. 568, 28-37. doi: 10.1016/j.abb.2015.01.008

Bowerman, A. F., Newberry, M., Dielen, A.-S., Whan, A., Larroque, O., Pritchard, J., et al. (2016). Suppression of glucan, water dikinase in the endosperm alters wheat grain properties, germination and coleoptile growth. Plant Biotechnol. J. 14, 398-408. doi: 10.1111/pbi.12394

Brummell, D. A., Watson, L. M., Zhou, J., McKenzie, M. J., Hallett, I. C., Simmons, L., et al. (2015). Overexpression of STARCH BRANCHING ENZYME II increases short-chain branching of amylopectin and alters the physicochemical properties of starch from potato tuber. BMC Biotechnol. 15:28. doi: 10.1186/s12896-015-0143-y

Cakir, B., Shiraishi, S., Tuncel, A., Matsusaka, H., Satoh, R., Singh, S., et al. (2016). Analysis of the rice ADPglucose transporter (OsBT1) indicates the presence of regulatory processes in the amyloplast stroma that control ADPglucose flux into starch. Plant Physiol. 170, 1271-1283. doi: 10.1104/ pp.15.01911

Chia, T., Thorneycroft, D., Chapple, A., Messerli, G., Chen, J., Zeeman, S. C., et al. (2004). A cytosolic glucosyltransferase is required for conversion of starch to sucrose in Arabidopsis leaves at night. Plant J. 37, 853-863. doi: 10.1111/j.1365-313X.2003.02012.x

Crozet, P., Margalha, L., Confraria, A., Rodrigues, A., Martinho, C., Adamo, M., et al. (2014). Mechanisms of regulation of SNF1/AMPK/SnRK1 protein kinases. Front. Plant Sci. 5. doi: 10.3389/fpls.2014.00190

Dobrenel, T., Caldana, C., Hanson, J., Robaglia, C., Vincentz, M., Veit, B., et al. (2016). TOR signaling and nutrient sensing. Annu. Rev. Plant Biol. 67, 261-285. doi: 10.1146/annurev-arplant-043014-114648

Edgerton, M. D. (2009). Increasing crop productivity to meet global needs for feed, food, and fuel. Plant Physiol. 149, 7-13. doi: 10.1104/pp.108.130195

Fodor, N., Challinor, A., Droutsas, I., Ramirez-Villegas, J., Zabel, F., Koehler, A.-K., et al. (2017). Integrating plant science and crop modeling: assessment of the impact of climate change on soybean and maize production. Plant Cell Physiol. 58, 1833-1847. doi: 10.1093/pcp/pcx141

Fu, F.-F., and Xue, H.-W. (2010). Coexpression analysis identifies rice starch regulator 1, a rice AP2/EREBP family transcription factor, as a novel rice starch biosynthesis regulator. Plant Physiol. 154, 927-938. doi: 10.1104/ pp.110.159517

Fu, Y., Ballicora, M. A., Leykam, J. F., and Preiss, J. (1998). Mechanism of reductive activation of potato tuber ADP-glucose pyrophosphorylase. J. Biol. Chem. 273, 25045-25052. doi: 10.1074/jbc.273.39.25045

Gámez-Arjona, F. M., Li, J., Raynaud, S., Baroja-Fernández, E., Muñoz, F. J., Ovecka, M., et al. (2011). Enhancing the expression of starch synthase class IV results in increased levels of both transitory and long-term storage starch. Plant Biotechnol. J. 9, 1049-1060. doi: 10.1111/j.1467-7652.2011.00626.x

Geigenberger, P. (2001). Tuber physiology and properties of starch from tubers of transgenic potato plants with altered plastidic adenylate transporter activity. Plant Physiol. 125, 1667-1678. doi: 10.1104/pp.125.4.1667

Geigenberger, P., Regierer, B., Nunes-Nesi, A., Leisse, A., Urbanczyk-Wochniak, E., Springer, F., et al. (2005). Inhibition of de novo pyrimidine synthesis in growing potato tubers leads to a compensatory stimulation of the pyrimidine salvage pathway and a subsequent increase in biosynthetic performance. Plant Cell 17, 2077-2088. doi: 10.1105/tpc.105.033548

Georgelis, N., and Hannah, L. C. (2008). Isolation of a heat-stable maize endosperm ADP-glucose pyrophosphorylase variant. Plant Sci. 175, 247-254. doi: 10.1016/j.plantsci.2008.04.005

Gibson, K., Park, J.-S., Nagai, Y., Hwang, S.-K., Cho, Y.-C., Roh, K.-H., et al. (2011). Exploiting leaf starch synthesis as a transient sink to elevate photosynthesis, plant productivity and yields. Plant Sci. 181, 275-281. doi: 10.1016/j.plantsci.2011.06.001

Glaring, M. A., Skryhan, K., Kötting, O., Zeeman, S. C., and Blennow, A. (2012). Comprehensive survey of redox sensitive starch metabolising enzymes in Arabidopsis thaliana. Plant Physiol. Biochem. 58, 89-97. doi: 10.1016/j. plaphy.2012.06.017

Gontarek, B. C., Neelakandan, A. K., Wu, H., and Becraft, P. W. (2016). NKD transcrition factors are central regulators of maize endosperm development. Plant Cell 28, 2916-2936. doi: 10.1105/tpc.16.00609

Griffiths, C. A., Sagar, R., Geng, Y., Primavesi, L. F., Patel, M. K., Passarelli, M. K., et al. (2016). Chemical intervention in plant sugar signalling increases yield and resilience. Nature 540, 574-578. doi: 10.1038/nature20591

Guan, S., Wang, P., Liu, H., Liu, G., Ma, Y., and Zhao, L. (2011). Production of high-amylose maize lines using RNA interference in sbe2a. African $J$. Biotechnol. 10, 15229-15237. doi: 10.5897/AJB11.943

Haedrich, N., Gibon, Y., Schudoma, C., Altmann, T., Lunn, J. E., and Stitt, M. (2011). Use of TILLING and robotised enzyme assays to generate an allelic series of Arabidopsis thaliana mutants with altered ADP-glucose pyrophosphorylase activity. J. Plant Physiol. 168, 1395-1405. doi: 10.1016/j.jplph.2011.01.013

Haedrich, N., Hendriks, J. H. M., Kötting, O., Arrivault, S., Feil, R., Zeeman, S. C., et al. (2012). Mutagenesis of cysteine 81 prevents dimerization of the APS1 subunit of ADP-glucose pyrophosphorylase and alters diurnal starch turnover in Arabidopsis thaliana leaves. Plant J. 70, 231-242. doi: 10.1111/j.1365-313X.2011.04860.x

Hannah, L. C., Futch, B., Bing, J., Shaw, J. R., Boehlein, S., Stewart, J. D., et al. (2012). A shrunken-2 transgene increases maize yield by acting in maternal tissues to increase the frequency of seed development. Plant Cell 24, 2352-2363. doi: $10.1105 /$ tpc.112.100602

Hannah, L. C., Shaw, J. R., Clancy, M. A., Georgelis, N., and Boehlein, S. K. (2017). A brittle-2 transgene increases maize yield by acting in maternal tissues to increase seed number. Plant Direct 1-9. doi: 10.1002/pld3.29

Jonik, C., Sonnewald, U., Hajirezaei, M.-R., Flügge, U.-I., and Ludewig, F. (2012). Simultaneous boosting of source and sink capacities doubles tuber starch yield of potato plants. Plant Biotechnol. J. 10, 1088-1098. doi: 10.1111/j.1467-7652.2012.00736.x

Kaplan, F., and Guy, C. L. (2004). Beta-amylase induction and the protective role of maltose during temperature shock. Plant Physiol. 135, 1674-1684. doi: 10.1104/pp.104.040808

Kaplan, F., Sung, D. Y., and Guy, C. L. (2006). Roles of beta-amylase and starch breakdown during temperatures stress. Physiol. Plant. 126, 120-128. doi: 10.1111/j.1399-3054.2006.00604.x

Kolbe, A., Tiessen, A., Schluepmann, H., Paul, M., Ulrich, S., and Geigenberger, P. (2005). Trehalose 6-phosphate regulates starch synthesis via posttranslational redox activation of ADP-glucose pyrophosphorylase. Proc. Natl. Acad. Sci. U. S. A. 102, 11118-11123. doi: 10.1073/pnas.0503410102

Kötting, O., Kossmann, J., Zeeman, S. C., and Lloyd, J. R. (2010). Regulation of starch metabolism: the age of enlightenment? Curr. Opin. Plant Biol. 13, 321-329. doi: 10.1016/j.pbi.2010.01.003

Li, T., Xu, S.-L., Oses-Prieto, J. A., Putil, S., Xu, P., Wang, R.-J., et al. (2011). Proteomics analysis reveals post-translational mechanisms for cold-induced metabolic changes in Arabidopsis. Mol. Plant 4, 361-374. doi: 10.1093/mp/ssq078

Liu, F., Zhao, Q., Mano, N., Ahmed, Z., Nitschke, F., Steup, M., et al. (2016). Modification of starch metabolism in transgenic Arabidopsis thaliana increases plant biomass and doubles oil seed production. Plant Biotechnol. J. 14, 976-985. doi: 10.1111/pbi.12453

Lloyd, J. R., and Kossmann, J. (2015). Transitory and storage starch metabolism: two sides of the same coin? Curr. Opin. Biotechnol. 32, 143-148. doi: 10.1016/j. copbio.2014.11.026

MacNeill, G. J., Mehrpouyan, S., Minow, M. A. A., Patterson, J. A., Tetlow, I. J., and Emes, M. J. (2017). Starch as a source, starch as a sink: the bifunctional role of starch in carbon allocation. J. Exp. Bot. 68, 4433-4453. doi: 10.1093/ jxb/erx291

Mahlow, S., Orzechowski, S., and Fettke, J. (2016). Starch phosphorylation: insights and perspectives. Cell. Mol. Life Sci. 73, 2753-2764. doi: 10.1007/ s00018-016-2248-4

Martins, M. C. M., Hejazi, M., Fettke, J., Steup, M., Feil, R., Krause, U., et al. (2013). Feedback inhibition of starch degradation in Arabidopsis leaves mediated by trehalose 6-phosphate. Plant Physiol. 163, 1142-1163. doi: $10.1104 /$ pp. 113.226787 
Matsoukas, I. G., Massiah, A. J., and Thomas, B. (2013). Starch metabolism and antiflorigenic signals modulate the juvenile-to-adult phase transition in Arabidopsis. Plant. Cell Environ. 36, 1802-1811. doi: 10.1111/pce.12088

McKibbin, R. S., Muttucumaru, N., Paul, M. J., Powers, S. J., Burrell, M. M., Coates, S., et al. (2006). Production of high-starch, low-glucose potatoes through over-expression of the metabolic regulator SnRK1. Plant Biotechnol. J. 4, 409-418. doi: 10.1111/j.1467-7652.2006.00190.x

Mikkelsen, R., Mutenda, K. E., Mant, A., Schurmann, P., and Blennow, A. (2005). Alpha-glucan, water dikinase (GWD): a plastidic enzyme with redoxregulated and coordinated catalytic activity and binding affinity. Proc. Natl. Acad. Sci. U. S. A. 102, 1785-1790. doi: 10.1073/pnas.0406674102

Nazarian-Firouzabadi, F., and Visser, R. G. F. (2017). Potato starch synthases: functions and relationships. Biochem. Biophys. Rep. 10, 7-16. doi: 10.1016/j. bbrep.2017.02.004

Nuccio, M. L., Wu, J., Mowers, R., Zhou, H.-P., Meghji, M., Primavesi, L. F., et al. (2015). Expression of trehalose-6-phosphate phosphatase in maize ears improves yield in well-watered and drought conditions. Nat. Biotechnol. 33, 862-869. doi: $10.1038 /$ nbt.3277

Oiestad, A. J., Martin, J. M., and Giroux, M. J. (2016). Overexpression of ADP-glucose pyrophosphorylase in both leaf and seed tissue synergistically increase biomass and seed number in rice (Oryza sativa ssp. japonica). Funct. Plant Biol. 43, 1194-1204. doi: 10.1071/FP16218

Paparelli, E., Parlanti, S., Gonzali, S., Novi, G., Mariotti, L., Ceccarelli, N., et al. (2013). Nighttime sugar starvation orchestrates gibberellin biosynthesis and plant growth in Arabidopsis. Plant Cell 25, 3760-3769. doi: 10.1105/tpc.113.115519

Pfister, B., and Zeeman, S. C. (2016). Formation of starch in plant cells. Cell. Mol. Life Sci. 73, 2781-2807. doi: 10.1007/s00018-016-2250-x

Ral, J.-P., Bowerman, A. F., Li, Z., Sirault, X., Furbank, R., Pritchard, J. R., et al. (2012). Down-regulation of glucan, water-dikinase activity in wheat endosperm increases vegetative biomass and yield. Plant Biotechnol. J. 10, 871-882. doi: 10.1111/j.1467-7652.2012.00711.x

Regierer, B., Fernie, A. R., Springer, F., Perez-Melis, A., Leisse, A., Koehl, K., et al. (2002). Starch content and yield increase as a result of altering adenylate pools in transgenic plants. Nat. Biotechnol. 20, 1256-1260. doi: 10.1038/nbt760

Rolland, F., Baena-Gonzalez, E., and Sheen, J. (2006). Sugar sensing and signaling in plants conserved and novel mechanisms. Annu. Rev. Plant Biol. 57, 675-709. doi: 10.1146/annurev.arplant.57.032905.105441

Ruckle, M., Bernasconi, L., Kölliker, R., Zeeman, S., and Studer, B. (2018). Genetic diversity of diurnal carbohydrate accumulation in white clover (Trifolium repens L.). Agronomy 8:47. doi: 10.3390/agronomy8040047

Ruckle, M., Meier, M., Frey, L., Eicke, S., Kölliker, R., Zeeman, S., et al. (2017). Diurnal leaf starch content: an orphan trait in forage legumes. Agronomy 7:16. doi: $10.3390 /$ agronomy7010016

Santelia, D., Trost, P., and Sparla, F. (2015). New insights into redox control of starch degradation. Curr. Opin. Plant Biol. 25, 1-9. doi: 10.1016/j.pbi.2015.04.003

Sanz-Barrio, R., Corral-Martinez, P., Ancin, M., Segui-Simarro, J. M., and Farran, I. (2013). Overexpression of plastidial thioredoxin fleads to enhanced starch accumulation in tobacco leaves. Plant Biotechnol. J. 11, 618-627. doi: 10.1111/pbi.12052

Schlosser, A. J., Martin, J. M., Beecher, B. S., and Giroux, M. J. (2014). Enhanced rice growth is conferred by increased leaf ADP-glucose pyrophosphorylase activity. J. Plant Physiol. Pathol. 2:4. doi: 10.4172/2329-955X.1000136

Sehnke, P. C., Chung, H.-J., Wu, K., and Ferl, R. J. (2001). Regulation of starch accumulation by granule-associated plant 14-3-3 proteins. Proc. Natl. Acad. Sci. U. S. A. 98, 765-770. doi: 10.1073/pnas.021304198

Seung, D., Thalmann, M., Sparla, F., Abou Hachem, M., Lee, S. K., IssakidisBourguet, E., et al. (2013). Arabidopsis thaliana AMY3 is a unique redoxregulated chloroplastic alpha-amylase. J. Biol. Chem. 288, 33620-33633. doi: 10.1074/jbc.M113.514794

Shaik, S. S., Carciofi, M., Martens, H. J., Hebelstrup, K. H., and Blennow, A. (2014). Starch bioengineering affects cereal grain germination and seedling establishment. J. Exp. Bot. 65, 2257-2270. doi: 10.1093/jxb/eru107

Shi, L., Wu, Y., and Sheen, J. (2018). TOR signaling in plants: conservation and innovation. Development 145:dev160887. doi: 10.1242/dev.160887

Skeffington, A. W., Graf, A., Duxbury, Z., Gruissem, W., and Smith, A. M. (2014). Glucan, water dikinase exerts little control over starch degradation in Arabidopsis leaves at night. Plant Physiol. 165, 866-879. doi: 10.1104/pp.114.237016

Skryhan, K., Gurrieri, L., Sparla, F., Trost, P., and Blennow, A. (2018). Redox regulation of starch metabolism. Front. Plant Sci. 9:1344. doi: 10.3389/ fpls.2018.01344
Smidansky, E. D., Clancy, M., Meyer, F. D., Lanning, S. P., Blake, N. K., Talbert, L. E., et al. (2002). Enhanced ADP-glucose pyrophosphorylase activity in wheat endosperm increases seed yield. Proc. Natl. Acad. Sci. U. S. A. 99, 1724-1729. doi: 10.1073/pnas.022635299

Smidansky, E. D., Martin, J. M., Hannah, L. C., Fischer, A. M., and Giroux, M. J. (2003). Seed yield and plant biomass increases in rice are conferred by deregulation of endosperm ADP-glucose pyrophosphorylase. Planta 216, 656-664. doi: 10.1007/s00425-002-0897-z

Sokolov, L. N., Dominguez-Solis, J. R., Allary, A. L., Buchanan, B. B., and Luan, S. (2006). A redox-regulated chloroplast protein phosphatase binds to starch diurnally and functions in its accumulation. Proc. Natl. Acad. Sci. U. S. A. 103, 9732-9737. doi: 10.1073/pnas.0603329103

Sonnewald, U., and Kossmann, J. (2013). Starches-from current models to genetic engineering. Plant Biotechnol. J. 11, 223-232. doi: 10.1111/pbi.12029

Stark, D. M., Timmerman, K. P., Barry, G. F., Preiss, J., and Kishore, G. M. (1992). Regulation of the amount of starch in plant tissues by ADP glucose pyrophosphorylase. Science 258, 287-292. doi: 10.1126/science.258.5080.287

Stitt, M., Gibon, Y., Lunn, J. E., and Piques, M. (2007). Multilevel genomics analysis of carbon signalling during low carbon availability: coordinating the supply and utilisation of carbon in a fluctuating environment. Funct. Plant Biol. 34, 526-549. doi: 10.1071/FP06249

Stitt, M., and Zeeman, S. C. (2012). Starch turnover: pathways, regulation and role in growth. Curr. Opin. Plant Biol. 15, 282-292. doi: 10.1016/j.pbi.2012.03.016

Streb, S., Eicke, S., and Zeeman, S. C. (2012). The simultaneous abolition of three starch hydrolases blocks transient starch breakdown in Arabidopsis. J. Biol. Chem. 287, 41745-41756. doi: 10.1074/jbc.M112.395244

Tetlow, I. J., and Emes, M. J. (2017). Starch biosynthesis in the developing endosperms of grasses and cereals. Agronomy 7:81. doi: 10.3390/ agronomy7040081

Thalmann, M., and Santelia, D. (2017). Starch as a determinant of plant fitness under abiotic stress. New Phytol. 214, 943-951. doi: 10.1111/nph.14491

Tiessen, A., Hendriks, J. H. M., Stitt, M., Branscheid, A., Gibon, Y., Farre, E. M., et al. (2002). Starch synthesis in potato tubers is regulated by posttranslational redox modification of ADP-glucose pyrophosphorylase: a novel regulatory mechanism linking starch synthesis to the sucrose supply. Plant Cell 14, 2191-2213. doi: 10.1105/tpc.003640

Tjaden, J., Mohlmann, T., Kampfenkel, K., Neuhaus, G. H., and Ekkehard Neuhaus, H. (1998). Altered plastidic ATP/ADP-transporter activity influences potato (Solanum tuberosum L.) tuber morphology, yield and composition of tuber starch. Plant J. 16, 531-540. doi: 10.1046/j.1365-313x.1998.00317.x

Tuncel, A., and Okita, T. W. (2013). Improving starch yield in cereals by overexpression of ADPglucose pyrophosphorylase: expectations and unanticipated outcomes. Plant Sci. 211, 52-60. doi: 10.1016/j.plantsci.2013.06.009

Valerio, C., Costa, A., Marri, L., Issakidis-Bourguet, E., Pupillo, P., Trost, P., et al. (2011). Thioredoxin-regulated $\beta$-amylase (BAM1) triggers diurnal starch degradation in guard cells, and in mesophyll cells under osmotic stress. J. Exp. Bot. 62, 545-555. doi: 10.1093/jxb/erq288

Van Harsselaar, J. K., Lorenz, J., Senning, M., Sonnewald, U., and Sonnewald, S. (2017). Genome-wide analysis of starch metabolism genes in potato (Solanum tuberosum L.). BMC Genom. 18:37. doi: 10.1186/s12864-016-3381-Z

Wang, F., Ren, G., Li, F., Wang, B., Yang, Y., Ma, X., et al. (2018). Overexpression of GmSnRK1, a sorbean sucrose non-fermenting-1 related protein kinase 1 gene, results in directional alteration tof carbohydrate metabolism in transgenic Arabidopsis. Biotechnol. Biotechnol. Equip. doi: 10.1080/13102818.2018.1469431

Wang, J.-C., Xu, H., Zhu, Y., Liu, Q.-Q., and Cai, X.-L. (2013). OsbZIP58, a basic leucine xipper transcrition factor, regulates starch biosynthesis in rice endosperm. J. Exp. Bot. 64, 3453-3466, doi: 10.1093/jxb/ert187

Wang, X., Chang, L., Tong, Z., Wang, D., Yin, Q., Wang, D., et al. (2016). Proteomics profiling reveals carbohydrate metabolic enzymes and 14-3-3 proteins play important roles for starch accumulation during Cassava root tuberization. Sci. Rep. 6:19643. doi: 10.1038/srep19643

Weise, S. E., Aung, K., Jarou, Z. J., Mehrshahi, P., Li, Z., Hardy, A. C., et al. (2012). Engineering starch accumulation by manipulation of phosphate metabolism of starch. Plant Biotechnol. J. 10, 545-554. doi: 10.1111/j.1467-7652.2012.00684.x

Whan, A., Verbyla, A., Mieog, J., Howitt, C., and Ral, J.-P. (2017). Transferring a biomass enhancement biotechnology from glasshouse to field: a case study on wheat GWD RNAi. Agronomy 7:82. doi: 10.3390/agronomy7040082

White-Gloria, C., Johnson, J. J., Marritt, K., Kataya, A., Vahab, A., and Moorhead, G. B. (2018). Protein kinases and phosphatases of the plastid and their 
potential role in starch metabolism. Front. Plant Sci. 9:1032. doi: 10.3389/ fpls.2018.01032

Wurzinger, B., Nukarinen, E., Nägele, T., Weckwerth, W., and Teige, M. (2018). The SnRK1 kinase as central mediator of energy signaling between different organelles. Plant Physiol. 176, 1085-1094. doi: 10.1104/pp.17.01404

Xiao, Q., Wang, Y., Du, J., Li, H., Wei, B., Wang, Y., et al. (2017). ZmMYB14 is an important transcription factor involved in the regulation of the activity of the ZmBT 1 promoter in starch biosynthesis in maize. FEBS J. 284, 3079-3099. doi: 10.1111/febs.14179

Xie, G., Li, Z., Ran, Q., Wang, H., and Zhang, J. (2018). Over-expression of mutated $Z m D A 1$ or $Z m D A R 1$ gene improves maize kernel yield by enhancing starch synthesis. Plant Biotechnol. J. 16, 234-244. doi: 10.1111/pbi.12763

Zeeman, S. C., Kossmann, J., and Smith, A. M. (2010). Starch: its metabolism, evolution, and biotechnological modification in plants. Annu. Rev. Plant Biol. 61, 209-234. doi: 10.1146/annurev-arplant-042809-112301

Zhang, L., Häusler, R. E., Greiten, C., Hajirezaei, M.-R., Haferkamp, I., Neuhaus, H. E., et al. (2008). Overriding the co-limiting import of carbon and energy into tuber amyloplasts increases the starch content and yield of transgenic potato plants. Plant Biotechnol. J. 6, 453-464. doi: 10.1111/j.1467-7652.2008.00332.x

Zhang, X. L., Myers, A. M., and James, M. G. (2005). Mutations affecting starch synthase III in arabidopsis alter leaf starch structure and increase the rate of starch synthesis. Plant Physiol. 138, 663-674. doi: 10.1104/pp.105.060319

Conflict of Interest Statement: The authors declare that the research was conducted in the absence of any commercial or financial relationships that could be construed as a potential conflict of interest.

Copyright (C) 2019 Lloyd and Kossmann. This is an open-access article distributed under the terms of the Creative Commons Attribution License (CC BY). The use, distribution or reproduction in other forums is permitted, provided the original author(s) and the copyright owner(s) are credited and that the original publication in this journal is cited, in accordance with accepted academic practice. No use, distribution or reproduction is permitted which does not comply with these terms. 